\title{
Supplements:
}

\section{Circulating Free Fatty Acid and Phospholipid Signature Predicts Early Rapid Kidney Function Decline in Patients with Type 1 Diabetes}

\section{Authors:}

Farsad Afshinnia*1, Thekkelnaycke M. Rajendiran ${ }^{2,3}$, Chenchen $\mathrm{He}^{1}$, Jaeman Byun ${ }^{1}$, Daniel Montemayor ${ }^{4,5}$, Manjula Darshi ${ }^{4,5}$, Jana Tumnova ${ }^{4,5}$, Jiwan Kim ${ }^{4,5}$, Christine P Limonte ${ }^{6,7}$, Rachel G. Miller ${ }^{8}$, Tina Costacou ${ }^{8}$, Trevor J. Orchard ${ }^{8}$, Tarunveer S. Ahluwalia ${ }^{9,10 a}$, Peter Rossing ${ }^{9,10 b}$, Janet K Snell-Bergeon ${ }^{11}$, Ian H. de Boer ${ }^{6,7,12}$, Loki Natarajan ${ }^{13}$, George

Michailidis $^{14}$, Kumar Sharma ${ }^{* 4,5}$, Subramaniam Pennathur ${ }^{\star 1,2,15}$

\section{Affiliations:}

${ }^{1}$ University of Michigan, Department of Internal Medicine-Nephrology, Ann Arbor, MI, USA 'University of Michigan, Michigan Regional Comprehensive Metabolomics Resource Core, Ann Arbor, MI, USA

${ }^{3}$ University of Michigan, Department of Pathology, Ann Arbor, MI, USA ${ }^{4}$ Division of Nephrology, UT Health Science Center San Antonio, San Antonio, TX, USA ${ }^{5}$ Center for Renal Precision Medicine, Division of Nephrology, Department of Medicine, University of Texas Health San Antonio, San Antonio, TX, USA ${ }^{6}$ Division of Nephrology, Department of Medicine, University of Washington, Seattle, WA, USA ${ }^{7}$ Kidney Research Institute, University of Washington, Seattle, WA, USA

${ }^{8}$ Department of Epidemiology, Graduate School of Public Health, University of Pittsburgh, Pittsburgh, PA, USA

${ }^{9}$ Steno Diabetes Center Copenhagen, Copenhagen, Denmark

${ }^{10 a}$ Department of Biology, The Bioinformatics Center, University of Copenhagen, Copenhagen, Denmark

${ }^{10 b}$ Department of Clinical Medicine, University of Copenhagen, Copenhagen, Denmark

${ }^{11}$ Barbara Davis Center for Diabetes, University of Colorado Anschutz Medical Campus, Aurora, CO, USA

${ }^{12}$ Puget Sound VA Healthcare System, Seattle, WA, USA

${ }^{13}$ Division of Biostatistics and Bioinformatics, Herbert Wertheim School of Public Health and Longevity Science, and Moores UCSD Cancer Center, La Jolla, CA, USA

${ }^{14}$ Department of Statistics and the Informatics Institute, University of Florida, Gainesville, FL, USA

${ }^{15}$ University of Michigan, Department of Molecular and Integrative Physiology, Ann Arbor, MI, USA

\section{* Address Correspondence}




\section{Procedures:}

Sample preparation and Mass Spectrometry: We applied previously published procedures for lipid extraction(6; 7; 9), using the modified Bligh-Dyer method.(10) In brief, we retrieved 50 $\mu \mathrm{L}$ of plasma and added water/methanol/dichloromethane at room temperature with 2:2:2 volume ratio, followed by spiking of internal standards: PC17:0/17:0, LPC 17:0, phosphatidylglycerol (PG) 17:0/17:0, PE 17:0/17:0, TAG 17:0/17:0/17:0, SM 18:1/17:0, and palmitate (C16-d9). After collection of the organic layer and drying under nitrogen, we reconstituted the samples in $100 \mu \mathrm{L}$ of Buffer B composed of acetonitrile/water/isopropyl alcohol $(10: 5: 85)$ and $10 \mathrm{mM}$ ammonium acetate (NH4OAc). The samples were further diluted to $5 \%$ sample/ 95\% Buffer B, and $5 \mu \mathrm{L}$ was injected using a TurbolonSpray lon Source into an AB Sciex Triple Quadrupole/QTRAP 6500+ mass spectrometer using a $1.8 \mu \mathrm{m}$-particle, $50 \mathrm{~mm} \times$ 2.1 mm Waters Acquity HSS T3 column (Waters Inc, Milford, MA) with the corresponding guard column in reverse phase. Buffer A for liquid chromatography was acetonitrile:water (40:60). The source voltage was $5500 \mathrm{~V}$, temperature was $550^{\circ} \mathrm{C}$, and current gas flow was 35, 50, and 60 psi. Declustering potential was $60 \mathrm{~V}$, and entrance potential was $10 \mathrm{~V}$ with collision energy at 15 V. We scanned in both positive and negative modes in MS1 and MS2 in multiple reaction monitoring (MRM) mode for 20 minutes. Abundant TAG isotopomers were combined into one lipid feature.

For quantification of $\mathrm{ACs}(11)$, we added $500 \mu \mathrm{L}$ of methanol/chloroform/water (8:1:1) and $1 \mu \mathrm{L}$ of $\mathrm{AC}$ mixed internal standards to $25 \mu \mathrm{L}$ of thawed plasma for lipid extraction. Then, the supernatant was dried under nitrogen, resuspended by $50 \mu \mathrm{L}$ of water/acetonitrile (95:5) mixed with $15 \mathrm{mM} \mathrm{NH}_{4} \mathrm{OAc}$. Five $\mu \mathrm{L}$ was injected into the Agilent 6410 Triple quadrupole tandem mass spectrometer (Agilent, Santa Clara, CA) for data acquisition in MRM mode as reported previously. Each lipid molecule was quantified using the authenticated internal standards with known concentrations and a calibration curve corresponding to each internal 
standard (sFig 2). Lipids of the DAG and CE classes were normalized and quantified using the TAG internal standard. The overall lower limit of detection was $<15 \mathrm{~nm} / \mathrm{L}$, which is significantly below the natural abundance of measured lipids in plasma. Lipids are stable metabolites which do not undergo degradation upon proper storage at $-80^{\circ} \mathrm{C}$.

Quality control: Investigators conducting the lipidomic study were blinded to the clinical data until after generating the lipidomic data and sharing the data with other team members. We randomized the samples into 6 different batches for mass spectrometry runs. A reference sample (pooled plasma) was run up to 7 times at the beginning of each batch to assess the reproducibility of the mass spectrometry. A pool of test samples was run at the beginning and then after every 7 to 10 samples in the lipidomic study, and after every 12 to 15 samples in AC study to assess stability of the runs over time, inter- and intra-assay coefficient of variation, and the batch-to-batch variability (sFig 3 and 4). 
sFig 1: The study cohort structure showing the distribution of participants with slow versus fast decliners of eGFR $\left(\mathrm{mL} / \mathrm{min} / 1.73 \mathrm{~m}^{2}\right)$ in the Training and Validation sets stratified by categories of eGFR at baseline. eGFR: estimated glomerular filtration rate; EDC, Epidemiology of Diabetes Complications study; CACT, Coronary Artery Calcification in Type 1 Diabetes study.

\begin{tabular}{|c|c|c|c|c|c|}
\hline & & \multicolumn{2}{|c|}{ Cohort=817 patients } & & \\
\hline & $\begin{array}{c}\text { Training set: } \\
n=407\end{array}$ & & & $\begin{array}{c}\text { Validation set: } \\
n=410\end{array}$ & \\
\hline eGFR $\geq 90$ & \multirow{3}{*}{$\begin{array}{c}\text { eGFR: } 60-89 \\
\text { Slow: } n=131 \\
\text { Fast: } n=29\end{array}$} & eGFR<60 & eGFR $\geq 90$ & \multirow{3}{*}{$\begin{array}{c}\text { eGFR: } 60-89 \\
\text { Slow: } n=129 \\
\text { Fast: } n=29\end{array}$} & eGFR<60 \\
\hline Slow: $n=139$ & & Slow: $n=14$ & Slow: $n=142$ & & Slow: $n=12$ \\
\hline Fast: $\mathrm{n}=83$ & & Fast: $\mathrm{n}=11$ & Fast: $\mathrm{n}=86$ & & Fast: $n=12$ \\
\hline $\begin{array}{c}\text { Steno: } \\
\text { Slow: } n=72\end{array}$ & $\begin{array}{c}\text { Steno: } \\
\text { Slow: } n=80\end{array}$ & $\begin{array}{c}\text { Steno: } \\
\text { Slow: } n=1\end{array}$ & $\begin{array}{c}\text { Steno: } \\
\text { Slow: } n=74\end{array}$ & $\begin{array}{c}\text { Steno: } \\
\text { Slow: } n=79\end{array}$ & $\begin{array}{c}\text { Steno: } \\
\text { Slow: } n=1\end{array}$ \\
\hline $\begin{array}{c}\text { Fast: } n=9 \\
\text { FDC. }\end{array}$ & $\begin{array}{l}\text { Fast: } n=25 \\
\text { EDC: }\end{array}$ & $\begin{array}{c}\text { Fast: } n=10 \\
\text { FDC. }\end{array}$ & $\begin{array}{c}\text { Fast: } n=13 \\
\text { FDC. }\end{array}$ & $\begin{array}{c}\text { Fast: } n=23 \\
\text { EDC: }\end{array}$ & $\begin{array}{c}\text { Fast: } n=11 \\
\text { FDC. }\end{array}$ \\
\hline Slow: $n=26$ & Slow: $n=12$ & Slow: $n=0$ & Slow: $n=25$ & Slow: $n=13$ & Slow: $n=0$ \\
\hline Fast: $n=27$ & \multirow{2}{*}{$\begin{array}{l}\text { Fast: } \mathrm{n}=3 \\
\text { CACT: }\end{array}$} & Fast: $\mathrm{n}=0$ & Fast: $n=29$ & \multirow{2}{*}{$\begin{array}{c}\text { Fast: } \mathrm{n}=4 \\
\text { CACT: }\end{array}$} & Fast: $n=0$ \\
\hline САСТ: & & САСТ: & САCT: & & САСТ: \\
\hline Slow: $n=41$ & Slow: $n=39$ & Slow: $n=13$ & Slow: $n=43$ & Slow: $n=37$ & Slow: $n=11$ \\
\hline Fast: $n=47$ & Fast: $n=1$ & Fast: $n=1$ & Fast: $n=44$ & Fast: $n=2$ & Fast: $n=1$ \\
\hline
\end{tabular}


sFig 2. Calibration curves of authenticated internal standards in plasma, with lower limit of quantification $<30 \mathrm{nmol} / \mathrm{L}$ and lower limit of detection $<15 \mathrm{nmol} / \mathrm{L}$.

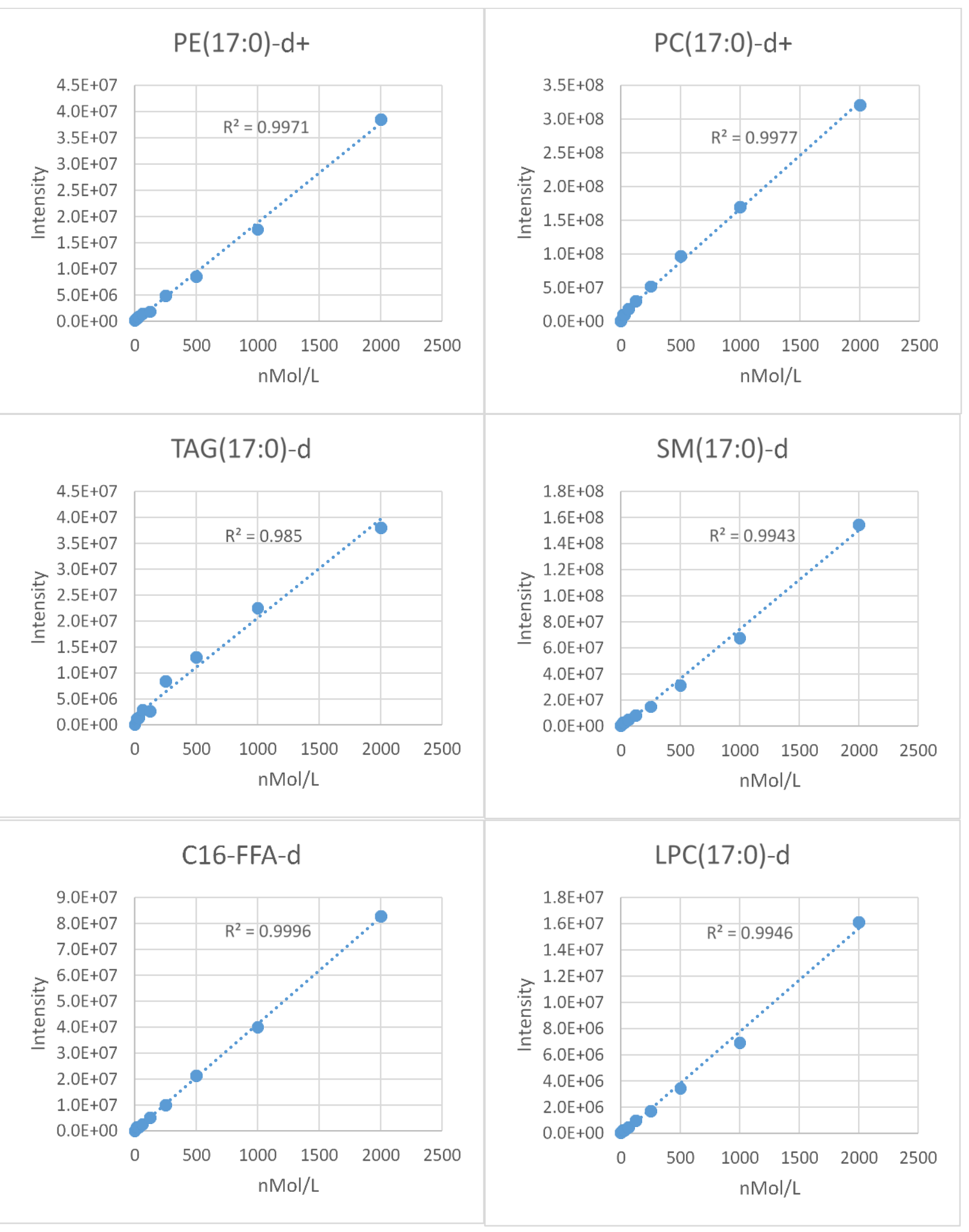


sFig 3. Comparing coefficients of variation (CV) of internal standards in test pools and pooled plasma in the Training and Validation sets.

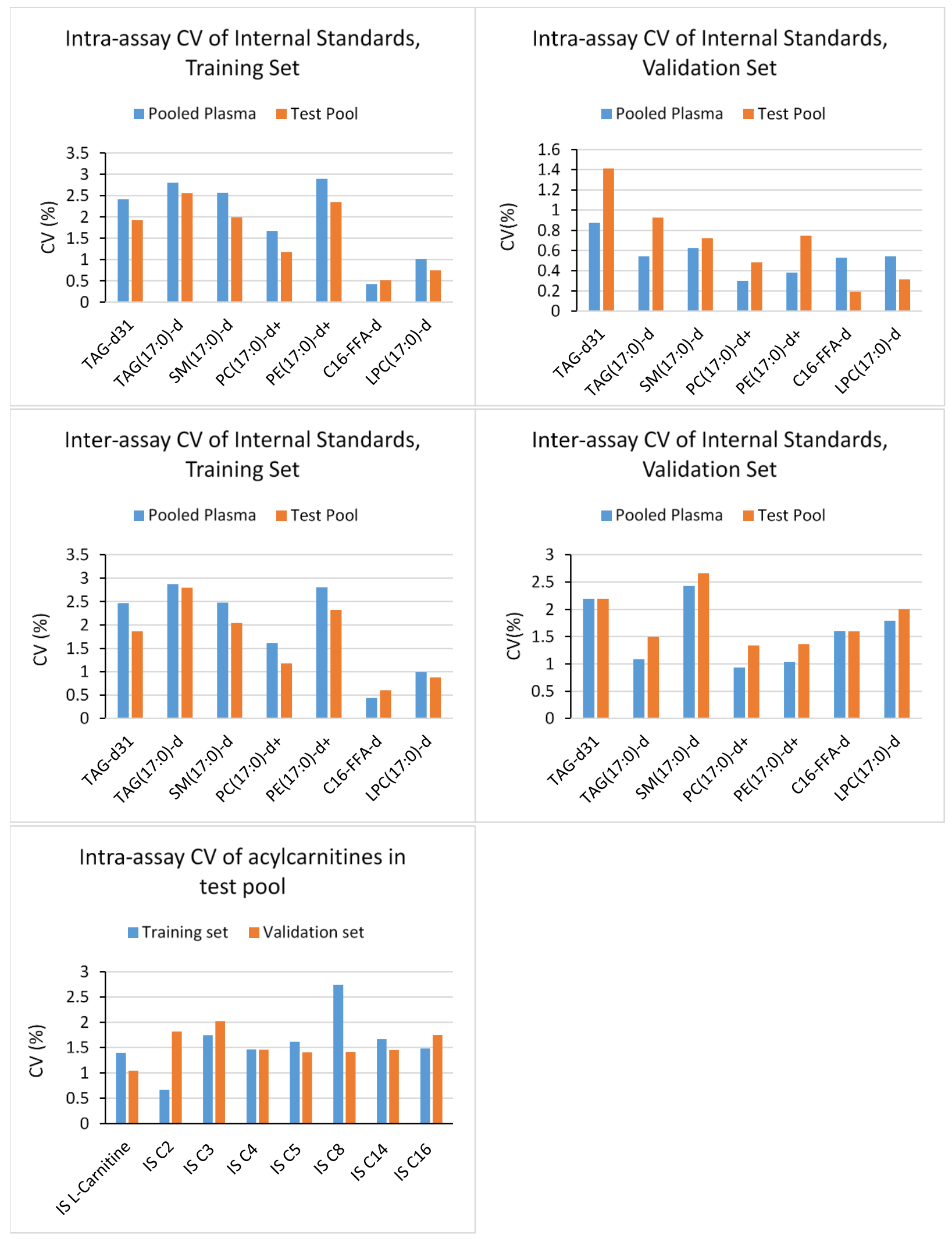


sFig 4. Illustration of minimal batch-to-batch variation.

A) Distribution of lipidomic platform internal standards in test pools and pooled plasma during sequential runs with the Training set
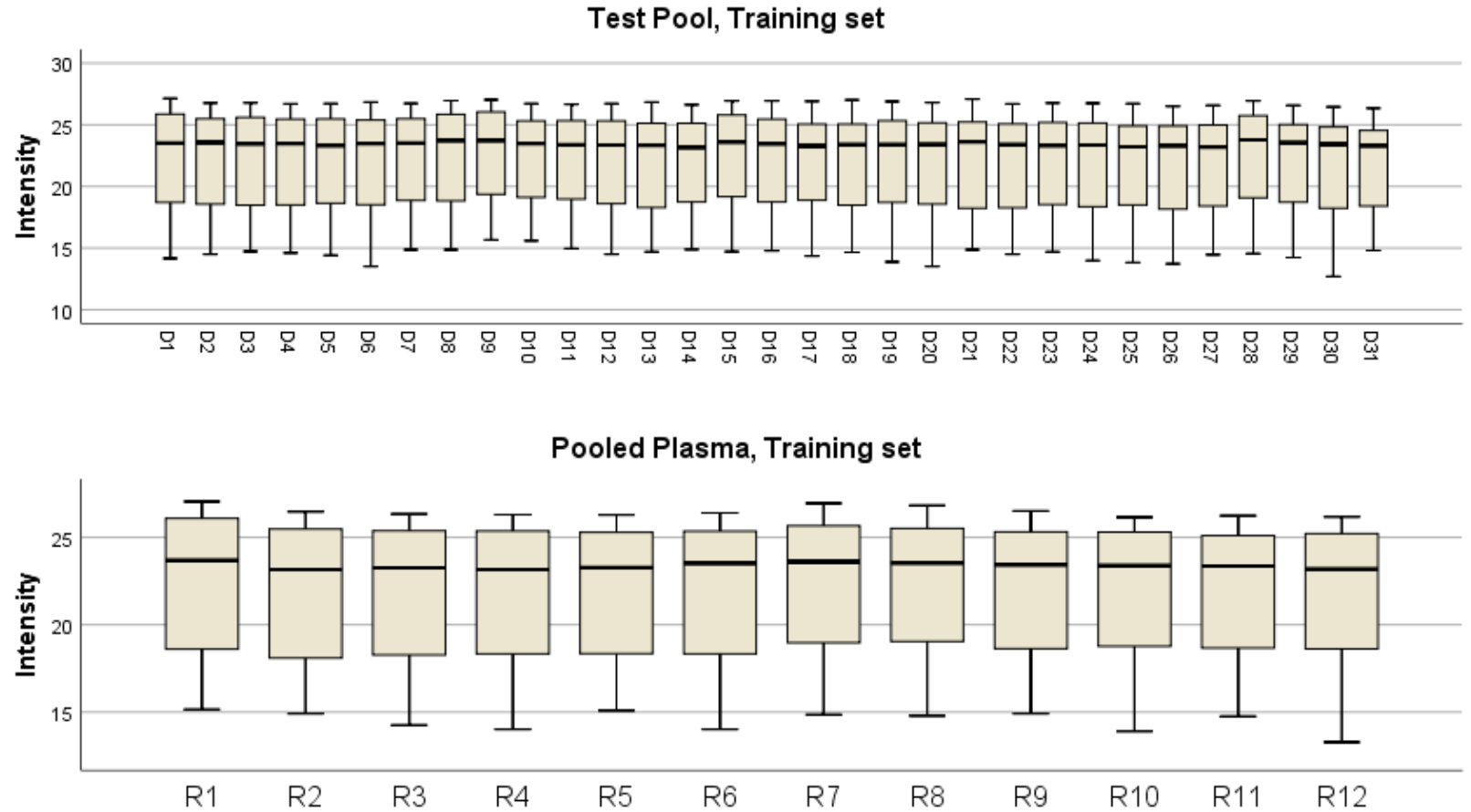

B) Distribution of lipidomic platform internal standards in test pools and pooled plasma in sequential runs with the Validation set

Test Pool, Validation Set

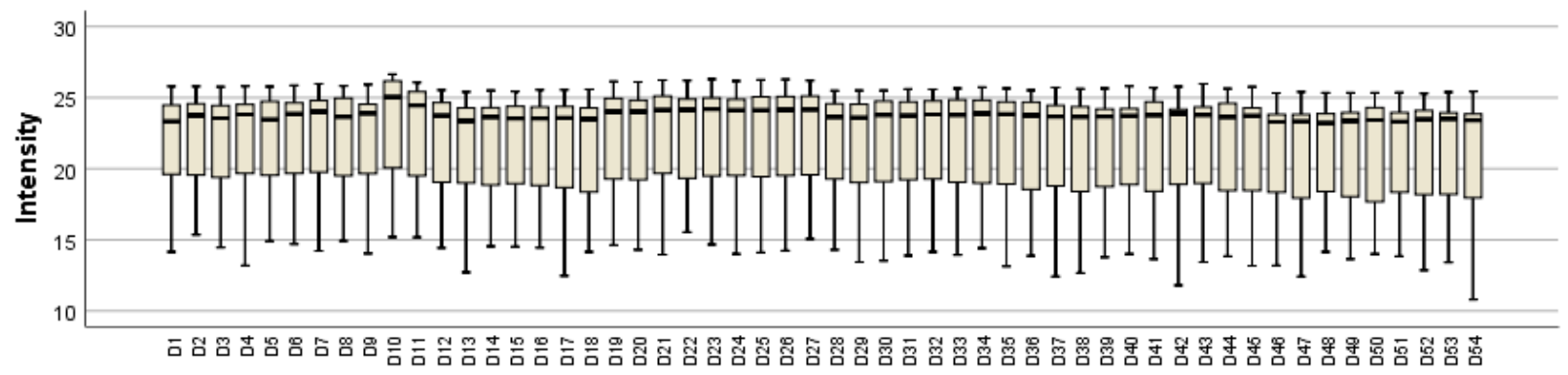

Pooled Plasma, Validation Set

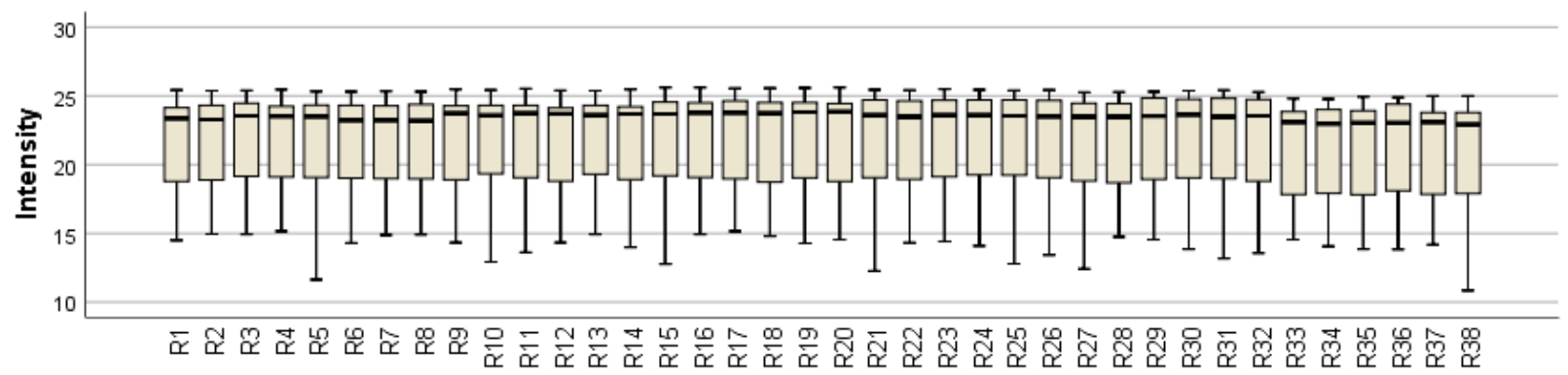


C) Distribution of endogenous acylcarnitines on a log2 scale in pooled plasma in sequential runs with the Training and Validation sets

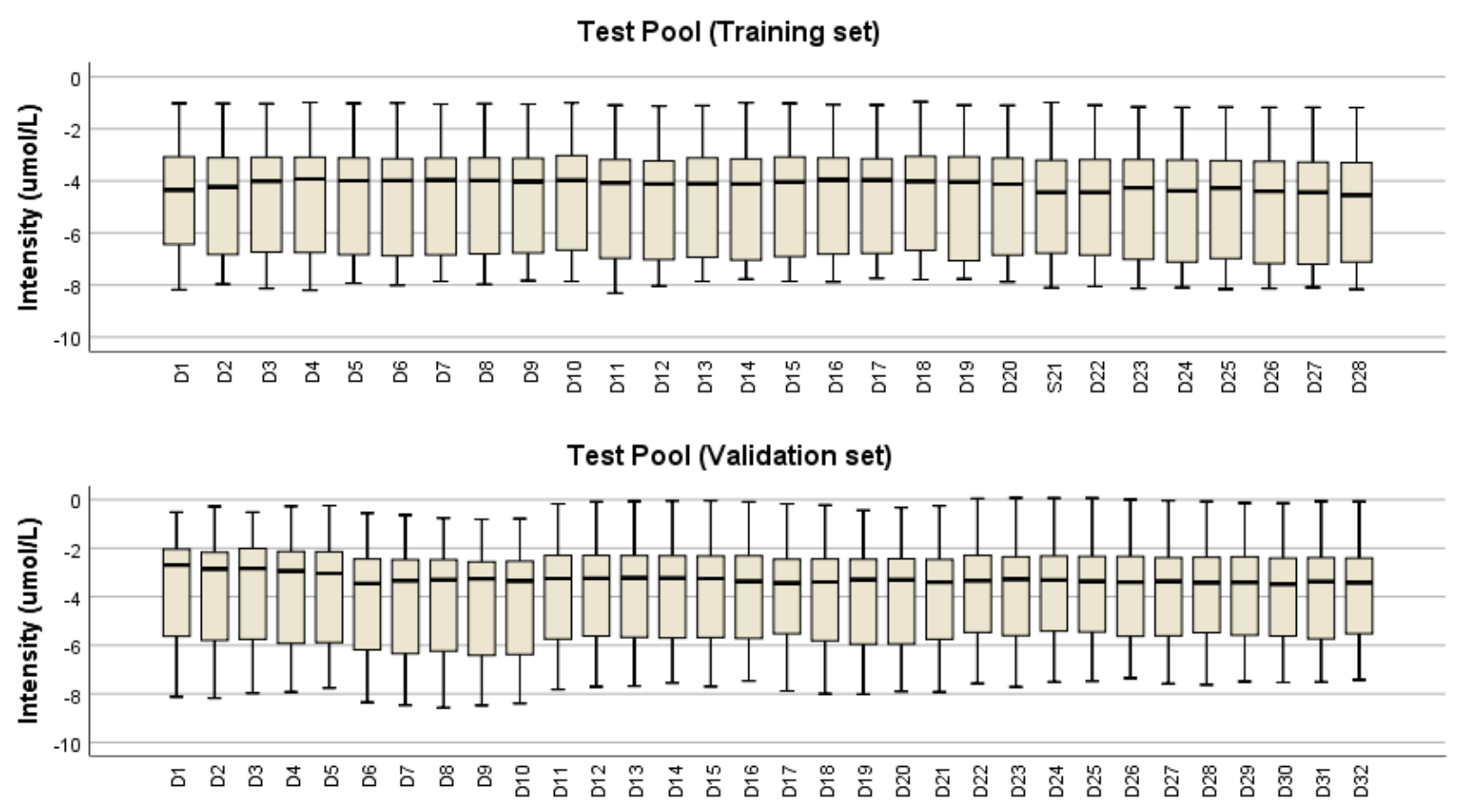


sFig 5. The top 47 lipids with differential levels in rapid versus slow eGFR decliners in the training set and validation set. Mean change is the standardized mean difference. Logistic regressions are unadjusted and adjusted univariate for each model. Adjustment was for age, sex, race, duration of diabetes, hypertension, use of angiotensin-converting-enzyme inhibitor or angiotensin receptor blocker, use of lipid lowering agents, and baseline mean arterial pressure, HbA1c, eGFR and urine albumin-creatinine ratio. AC, acylcarnitine; $\mathrm{CE}$, cholesteryl-ester; $\mathrm{Cl}$, confidence interval; FDR, false discovery rate; FFA, free fatty acid; LR, logistic regression; $\mathrm{OR}$, odds ratio; PC, phosphatidylcholine; PE, phosphatidylethanolamine; TAG, triacylglycerol. 


\begin{tabular}{|c|c|c|c|c|c|c|c|c|c|c|c|c|c|c|c|c|c|}
\hline \multirow[b]{2}{*}{ Lipids } & \multicolumn{3}{|c|}{ Training } & \multicolumn{3}{|c|}{ Validation } & \multicolumn{3}{|c|}{$\begin{array}{l}\text { Unadjusted LR, } \\
\text { Training set }\end{array}$} & \multicolumn{3}{|c|}{$\begin{array}{l}\text { Adjusted LR, } \\
\text { Training set }\end{array}$} & \multicolumn{3}{|c|}{$\begin{array}{l}\text { Unadjusted LR, } \\
\text { validation set }\end{array}$} & \multicolumn{2}{|c|}{$\begin{array}{l}\text { Adjusted } L R \text {, } \\
\text { validation set }\end{array}$} \\
\hline & \begin{tabular}{|c|} 
Mean \\
Change
\end{tabular} & $p$ & FDR & $\begin{array}{c}\text { Mean } \\
\text { Change } \\
\end{array}$ & $p$ & FDR & OR (95\% & $\% \mathrm{Cl}$ & $p$ & OR (95\% & & $p$ & OR (95\%) & $\% \mathrm{Cl})$ & $p$ & OR $(95 \% \mathrm{Cl})$ & $\mathrm{p}$ \\
\hline FFA(20:2) & 0.51 & $<0.0001$ & 0.001 & 0.39 & 0.0002 & 0.002 & & เ•r & $<0.0001$ & & $\bullet \bullet-1$ & 0.001 & & $1 \bullet-1$ & 0.0003 & 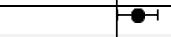 & 0.042 \\
\hline $\mathrm{CE}(22: 6)$ & -0.46 & $<0.0001$ & 0.001 & -0.37 & 0.0005 & 0.004 & t-r & & $<0.0001$ & $\mapsto \bullet$ & & 0.053 & $\mapsto-1$ & & 0.0007 & + & 0.356 \\
\hline TAG(58:8) & -0.46 & $<0.0001$ & 0.001 & -0.42 & 0.0001 & 0.001 & $1 \bullet-1$ & & $<0.0001$ & $\vdash \bullet-1$ & & 0.015 & $\vdash \bullet-1$ & & 0.0001 & $\mapsto-1$ & 0.012 \\
\hline $\mathrm{PE}(\mathrm{P}-18: 0 / 20: 5)$ & -0.47 & $<0.0001$ & 0.001 & -0.48 & $<0.0001$ & 0.000 & เ• & & $<0.0001$ & $\bullet-1$ & & 0.008 & $\mapsto \bullet$ & & $<0.0001$ & $\vdash \bullet-1$ & 0.007 \\
\hline FFA(24:3) & 0.44 & $<0.0001$ & 0.002 & 0.46 & $<0.0001$ & 0.001 & & •๑- & 0.0001 & & $1 \bullet-1$ & 0.005 & & $1 \bullet-1$ & $<0.0001$ & $1 \bullet-1$ & 0.006 \\
\hline FFA(18:2) & 0.44 & $<0.0001$ & 0.002 & 0.45 & $<0.0001$ & 0.001 & & $1 \bullet$ & 0.0001 & & $\mapsto \bullet-1$ & 0.004 & & $\vdash \bullet-1$ & $<0.0001$ & $-\rightarrow-1$ & 0.022 \\
\hline $\mathrm{CE}(20: 5)$ & -0.43 & $<0.0001$ & 0.002 & -0.52 & $<0.0001$ & 0.000 & $\mapsto$ & & 0.0001 & $\mapsto-1$ & & 0.011 & $\mapsto-1$ & & $<0.0001$ & $\mapsto-1$ & 0.004 \\
\hline $\operatorname{TAG}(58: 9)$ & -0.44 & $<0.0001$ & 0.002 & -0.37 & 0.0005 & 0.004 & $\mapsto-1$ & & 0.0001 & $\mapsto \bullet-1$ & & 0.012 & $r \bullet-1$ & & 0.0007 & & 0.141 \\
\hline TAG(60:12) & -0.44 & $<0.0001$ & 0.002 & -0.41 & 0.0001 & 0.002 & 10 & & 0.0001 & $\mapsto \bullet-1$ & & 0.006 & $1 \bullet-1$ & & 0.0002 & $\mapsto$ & 0.187 \\
\hline $\mathrm{PC}(16: 0 / 22: 6)$ & -0.45 & $<0.0001$ & 0.002 & -0.48 & $<0.0001$ & 0.000 & 1-1 & & $<0.0001$ & $\bullet-1$ & & 0.004 & $\mapsto-$ & & $<0.0001$ & $\mapsto \bullet-1$ & 0.005 \\
\hline $\mathrm{PC}(18: 2 / 20: 5)$ & -0.43 & $<0.0001$ & 0.002 & -0.35 & 0.0010 & 0.007 & $\bullet-1$ & & 0.0001 & $\bullet$ & & 0.188 & $1 \bullet-1$ & & 0.0012 & -1 & 0.442 \\
\hline $\mathrm{PC}(18: 0 / 20: 0)$ & -0.43 & $<0.0001$ & 0.002 & -0.37 & 0.0005 & 0.004 & $\mapsto-1$ & & 0.0001 & $\mapsto-1$ & & 0.008 & $-\bullet-1$ & & 0.0007 & $1 \bullet$ & 0.091 \\
\hline $\mathrm{PE}(18: 0 / 20: 5)$ & -0.43 & $<0.0002$ & 0.002 & -0.45 & $<0.0001$ & 0.001 & 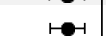 & & 0.0001 & $\mapsto$ & & 0.233 & $\vdash \bullet-1$ & & $<0.0001$ & & 0.274 \\
\hline AC3:0 & -0.41 & $<0.0002$ & 0.003 & -0.29 & 0.0063 & 0.029 & $\mapsto-1$ & & 0.0003 & $\mapsto-1$ & & 0.032 & $\mapsto-1$ & & 0.0071 & & 0.201 \\
\hline $\mathrm{PE}(\mathrm{P}-18: 1 / 20: 5)$ & -0.41 & $<0.0002$ & 0.003 & -0.49 & $<0.0001$ & 0.000 & $\vdash \bullet$ & & 0.0002 & $\mapsto \bullet-$ & & 0.013 & $\vdash \bullet$ & & $<0.0001$ & $\mapsto$ & 0.007 \\
\hline $\mathrm{PC}(18: 0 / 22: 6)$ & -0.41 & $<0.0002$ & 0.003 & -0.40 & 0.0002 & 0.002 & $\mapsto \bullet$ & & 0.0002 & $1 \bullet-1$ & & 0.008 & $1 \bullet-1$ & & 0.0003 & & 0.521 \\
\hline $\mathrm{PC}(16: 0 / 20: 5)$ & -0.41 & $<0.0002$ & 0.003 & -0.47 & $<0.0001$ & 0.000 & -૭ & & 0.0002 & $\mapsto \bullet-1$ & & 0.014 & $\vdash-1$ & & $<0.0001$ & $\vdash \bullet$ & 0.007 \\
\hline $\operatorname{TAG}(60: 11)$ & -0.41 & $<0.0002$ & 0.003 & -0.41 & 0.0001 & 0.001 & $\bullet \bullet$ & & 0.0002 & $\mapsto \bullet$ & & 0.143 & $\vdash \bullet-1$ & & 0.0002 & & 0.187 \\
\hline $\operatorname{TAG}(60: 10)$ & -0.40 & $<0.0002$ & 0.003 & -0.37 & 0.0005 & 0.004 & $1-1$ & & 0.0003 & $\mapsto$ & & 0.180 & $-\bullet$ & & 0.0006 & & 0.266 \\
\hline TAG(58:10) & -0.40 & $<0.0002$ & 0.003 & -0.34 & 0.0015 & 0.009 & $1 \bullet-1$ & & 0.0003 & $\mapsto \bullet$ & & 0.064 & $1 \bullet-1$ & & 0.0018 & & 0.250 \\
\hline $\mathrm{PC}(18: 0 / 20: 5)$ & -0.39 & 0.0003 & 0.004 & -0.52 & $<0.0001$ & 0.000 & $1-1$ & & $<0.0001$ & $\bullet \bullet$ & & 0.088 & $\mapsto \bullet$ & & $<0.0001$ & $\bullet$ & 0.020 \\
\hline $\operatorname{PEP}(16: 0 / 20: 5)$ & -0.39 & 0.0003 & 0.004 & -0.45 & $<0.0001$ & 0.001 & $\rightarrow$ & & $<0.0001$ & $\mapsto$ & & 0.197 & $\bullet-$ & & $<0.0001$ & $\mapsto$ & 0.110 \\
\hline $\mathrm{FFA}(24: 2)$ & 0.39 & 0.0003 & 0.004 & 0.36 & 0.0006 & 0.005 & & $1 \bullet-1$ & $<0.0001$ & & $\bullet-1$ & 0.013 & & $1 \bullet-1$ & 0.0010 & -1 & 0.104 \\
\hline TAG(56:8) & -0.38 & 0.0004 & 0.004 & -0.34 & 0.0012 & 0.008 & $-\bullet$ & & $<0.0001$ & $\vdash \bullet$ & & 0.108 & $\mapsto-1$ & & 0.0020 & & 0.185 \\
\hline $\mathrm{PC}(18: 1 / 20: 5)$ & -0.38 & 0.0004 & 0.005 & -0.41 & 0.0001 & 0.001 & $\vdash \bullet-1$ & & 0.0010 & $\mapsto$ & & 0.278 & $1 \bullet-1$ & & $<0.0001$ & & 0.113 \\
\hline TAG(58:7) & -0.38 & 0.0004 & 0.005 & -0.42 & 0.0001 & 0.001 & $1 \bullet-1$ & & 0.0010 & $\vdash \bullet$ & & 0.277 & $1 \bullet-1$ & & $<0.0001$ & $\mapsto$ & 0.036 \\
\hline $\operatorname{LPC}(22: 4)$ & 0.37 & 0.0005 & 0.005 & 0.31 & 0.0039 & 0.019 & & $1-1$ & 0.0010 & & $1 \bullet-1$ & 0.010 & & $1-1$ & 0.0040 & $\bullet-1$ & 0.109 \\
\hline TAG(56:7) & -0.37 & 0.0006 & 0.006 & -0.37 & 0.0005 & 0.004 & $1 \bullet-1$ & & 0.0010 & $\vdash \bullet$ & & 0.274 & $\bullet \bullet-1$ & & 0.0010 & & 0.151 \\
\hline TAG(54:8) & -0.36 & 0.0008 & 0.008 & -0.30 & 0.0051 & 0.024 & $-\rightarrow$ & & 0.0010 & $\mapsto \bullet$ & & 0.111 & $\vdash-1$ & & 0.0060 & & 0.261 \\
\hline $\mathrm{PE}(\mathrm{P}-18: 0 / 22: 6)$ & -0.35 & 0.0009 & 0.009 & -0.32 & 0.0025 & 0.014 & $1 \bullet-1$ & & 0.0010 & $\vdash \bullet$ & & 0.131 & $1 \bullet-1$ & & 0.0030 & & 0.471 \\
\hline TAG(56:9) & -0.35 & 0.0010 & 0.009 & -0.34 & 0.0014 & 0.009 & $-\bullet$ & & 0.0010 & $\mapsto \bullet$ & & 0.148 & $1 \bullet-1$ & & 0.0020 & $\mapsto$ & 0.150 \\
\hline $\operatorname{LPC}(20: 5)$ & -0.35 & 0.0010 & 0.009 & -0.44 & $<0.0001$ & 0.001 & $1 \bullet-1$ & & 0.0010 & $\mapsto \bullet$ & | & 0.289 & t-r & & $<0.0001$ & $\vdash$ & 0.233 \\
\hline FFA(18:1) & 0.35 & 0.0013 & 0.010 & 0.36 & 0.0006 & 0.005 & & $1 \bullet-1$ & 0.0020 & & $1 \bullet-1$ & 0.019 & & $1 \bullet-1$ & 0.0010 & -1 & 0.111 \\
\hline $\mathrm{FFA}(18: 0)$ & -0.34 & 0.0014 & 0.011 & -0.42 & 0.0001 & 0.001 & $-\bullet$ & & 0.0020 & $\mapsto-$ & & 0.044 & $\mapsto-1$ & & $<0.0001$ & $\vdash \bullet$ & 0.080 \\
\hline $\mathrm{PE}(18: 0 / 22: 6)$ & -0.33 & 0.0021 & 0.015 & -0.28 & 0.0090 & 0.037 & -1 & & 0.0030 & $\vdash \bullet$ & & 0.102 & $\bullet-1$ & & 0.0100 & $\mapsto \bullet$ & 0.076 \\
\hline $\operatorname{PE}(18: 1 / 16: 1)$ & -0.31 & 0.0038 & 0.024 & -0.30 & 0.0054 & 0.025 & $1 \bullet-1$ & & 0.0050 & $\mapsto \bullet$ & & 0.085 & $\vdash \bullet$ & & 0.0060 & $\vdash \bullet$ & 0.080 \\
\hline $\mathrm{PE}(\mathrm{P}-18: 1 / 22: 4)$ & 0.31 & 0.0038 & 0.024 & 0.42 & 0.0001 & 0.001 & & $1-1$ & 0.0040 & & & 0.456 & & $1 \bullet-1$ & $<0.0001$ & -1 & 0.151 \\
\hline TAG(58:6) & -0.30 & 0.0046 & 0.028 & -0.32 & 0.0029 & 0.015 & $1 \bullet-1$ & & 0.0060 & $\mapsto$ & & 0.483 & $1 \bullet-1$ & & 0.0040 & $\vdash$ & 0.077 \\
\hline $\mathrm{PE}(\mathrm{O}-16: 0 / 22: 6)$ & -0.30 & 0.0048 & 0.028 & -0.32 & 0.0025 & 0.014 & $-\infty$ & & 0.0060 & $\vdash \bullet$ & & 0.139 & $1 \bullet-1$ & & 0.0030 & $\mapsto$ & 0.200 \\
\hline $\mathrm{SM}(\mathrm{d} 18: 1 / 24: 1)$ & -0.30 & 0.0056 & 0.032 & -0.44 & $<0.0001$ & 0.001 & $1 \bullet-1$ & & 0.0060 & $\vdash \bullet$ & & 0.153 & $r \bullet$ & & $<0.0001$ & $\mapsto$ & 0.074 \\
\hline $\operatorname{LPC}(22: 6)$ & -0.30 & 0.0058 & 0.032 & -0.34 & 0.0013 & 0.008 & $1-1$ & & 0.0060 & $\vdash$ & & 0.445 & $1 \bullet-1$ & & 0.0020 & $\mapsto-1$ & 0.362 \\
\hline $\mathrm{PE}(\mathrm{O}-16: 0 / 20: 5)$ & -0.30 & 0.0058 & 0.032 & -0.38 & 0.0004 & 0.004 & $\bullet \bullet-1$ & & 0.0060 & $\vdash \bullet$ & & 0.232 & $\mapsto-1$ & & $<0.0001$ & $\mapsto-1$ & 0.331 \\
\hline $\mathrm{PE}(\mathrm{P}-16: 0 / 22: 6)$ & -0.30 & 0.0059 & 0.032 & -0.32 & 0.0026 & 0.014 & $1-1$ & & 0.0070 & $\mapsto$ & & 0.340 & $1 \bullet-1$ & & 0.0030 & $\mapsto$ & 0.299 \\
\hline $\mathrm{PC}(18: 1 / 22: 4)$ & 0.29 & 0.0066 & 0.035 & 0.34 & 0.0015 & 0.009 & & |•-1 & 0.0070 & & $\bullet$ & 0.077 & & $1-1$ & 0.0020 & - & 0.173 \\
\hline $\mathrm{PE}(18: 0 / 20: 1)$ & -0.29 & 0.0065 & 0.035 & -0.39 & 0.0003 & 0.003 & $1 \bullet-1$ & & 0.0070 & $\mapsto$ & & 0.307 & $\mapsto \bullet-1$ & & $<0.0001$ & $\mapsto \bullet$ & 0.211 \\
\hline TAG(54:7) & -0.29 & 0.0070 & 0.036 & -0.27 & 0.0102 & 0.039 & $\mapsto-1$ & & 0.0080 & $\vdash$ & & 0.310 & $\vdash-1$ & & 0.0110 & $\mapsto$ & 0.148 \\
\hline $\mathrm{PE}(16: 0 / 22: 6)$ & -0.28 & 0.0085 & 0.041 & -0.26 & 0.0138 & 0.049 & $\vdash \bullet$ & & 010000 & $\vdash \bullet$ & & 0.229 & $\mapsto \bullet-1$ & & 0.0150 & $\bullet$ & 0.193 \\
\hline
\end{tabular}


sFig 6. Constituents and their Pearson correlation coefficients with the corresponding principal component. AC, acylcarnitine; FFA: free fatty acid; PC: phosphatidylcholine; PE; phosphatidylethanolamine; PE-P, PE with alkenyl ether substitute at sn-1 carbon; PE-O, PE with alkyl ether substitute at sn-1 carbon of the glycerol backbone; SM: sphingomyelin; TAG: triacylglycerol.

A) Training set

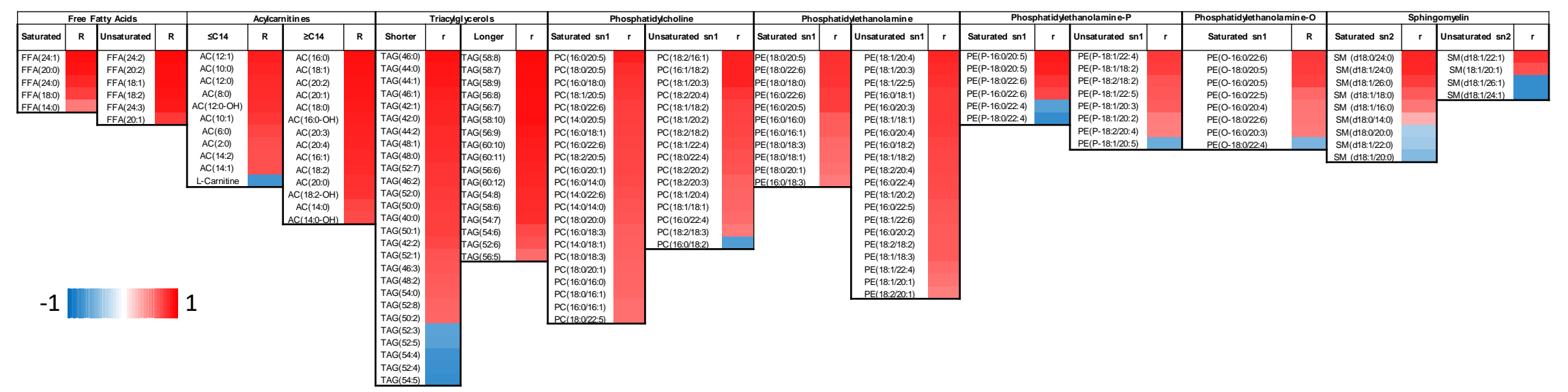

B) Validation set

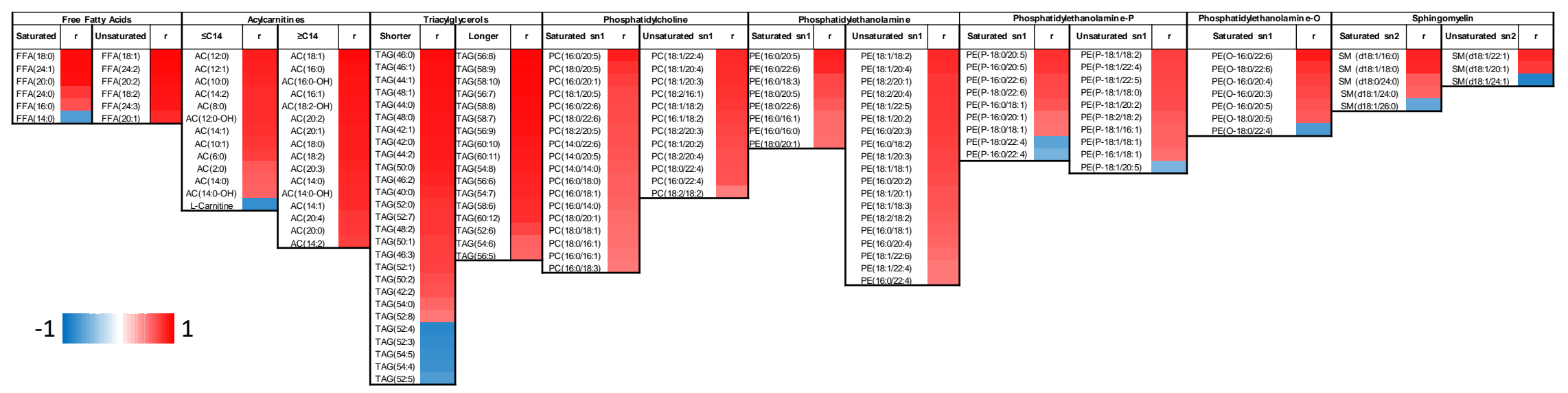


sFig 7. Comparing principal components representative of free fatty acid (FFA), acylcarnitines (AC), triacylglycerols (TAG), phosphatidylcholines (PC), and phosphatidylethanolamines (PE) in rapid versus slow decliners of eGFR stratified by eGFR categories in the validation set. Trend $p$ values of lipids by eGFR categories were 0.002 for shorter TAGs, 0.034 for longer TAGs, and $<0.001$ for other phospholipids in rapid decliners.

A) Saturated FFA

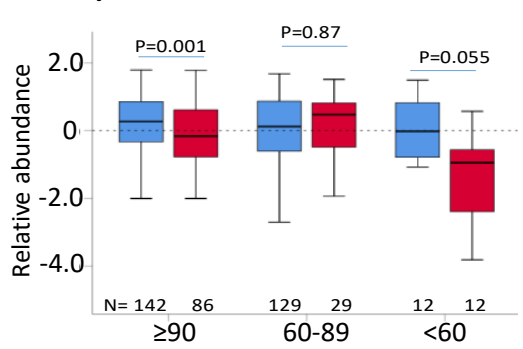

D) C14 and higher AC

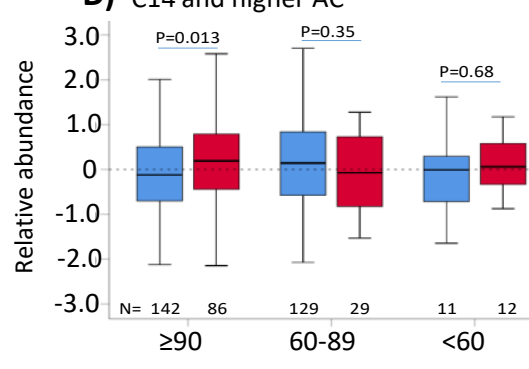

G) Saturated sn1 PC

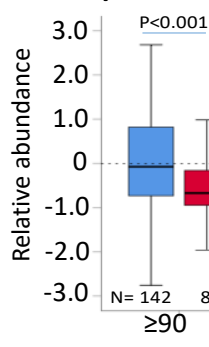

B) Unsaturated FFA

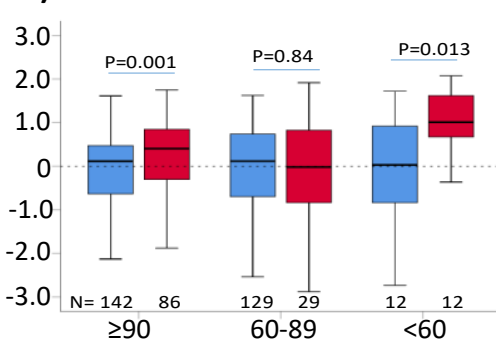

E) Shorter TAGs

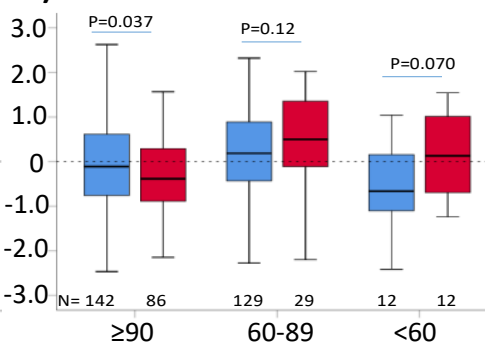

H) Unsaturated sn1 PC
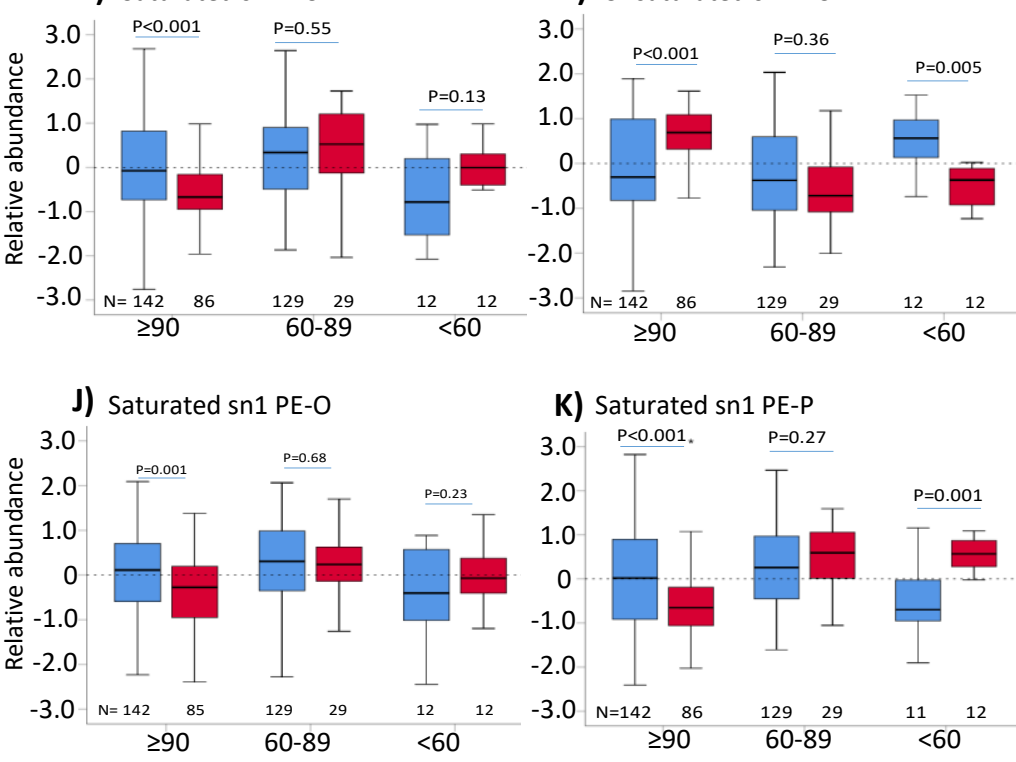

K) Saturated sn1 PE-P

K) Saturated sn1 PE-P
$P<0.001 . \quad P=0.27$

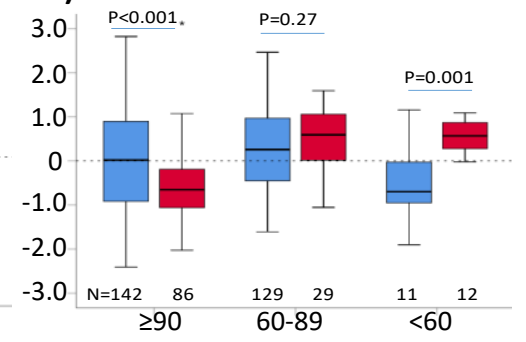

L) Unsaturated sn1 PE-P

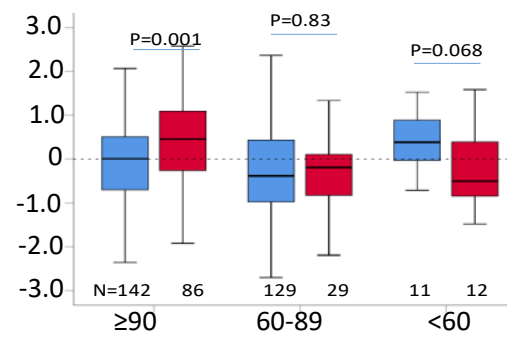

eGFR Categories $\left(\mathrm{mL} / \mathrm{min} / 1.73 \mathrm{~m}^{2}\right)$

C) C12 and shorter AC

Rapid

Decline

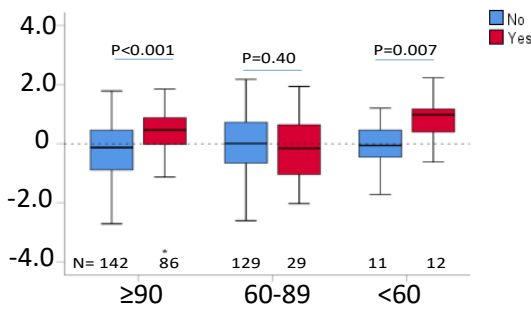

F) Longer TAGs

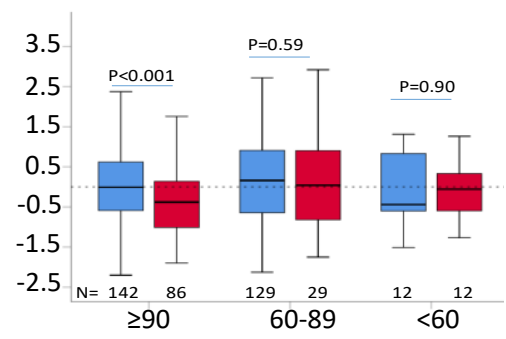

I) Saturated sn1 PE

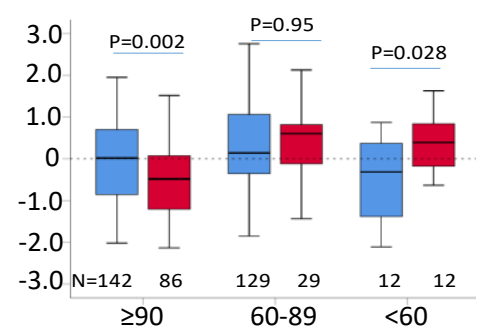




\section{A) eGFR $\geq 90 \mathrm{~mL} / \mathrm{min} / 1.73 \mathrm{~m} 2$}
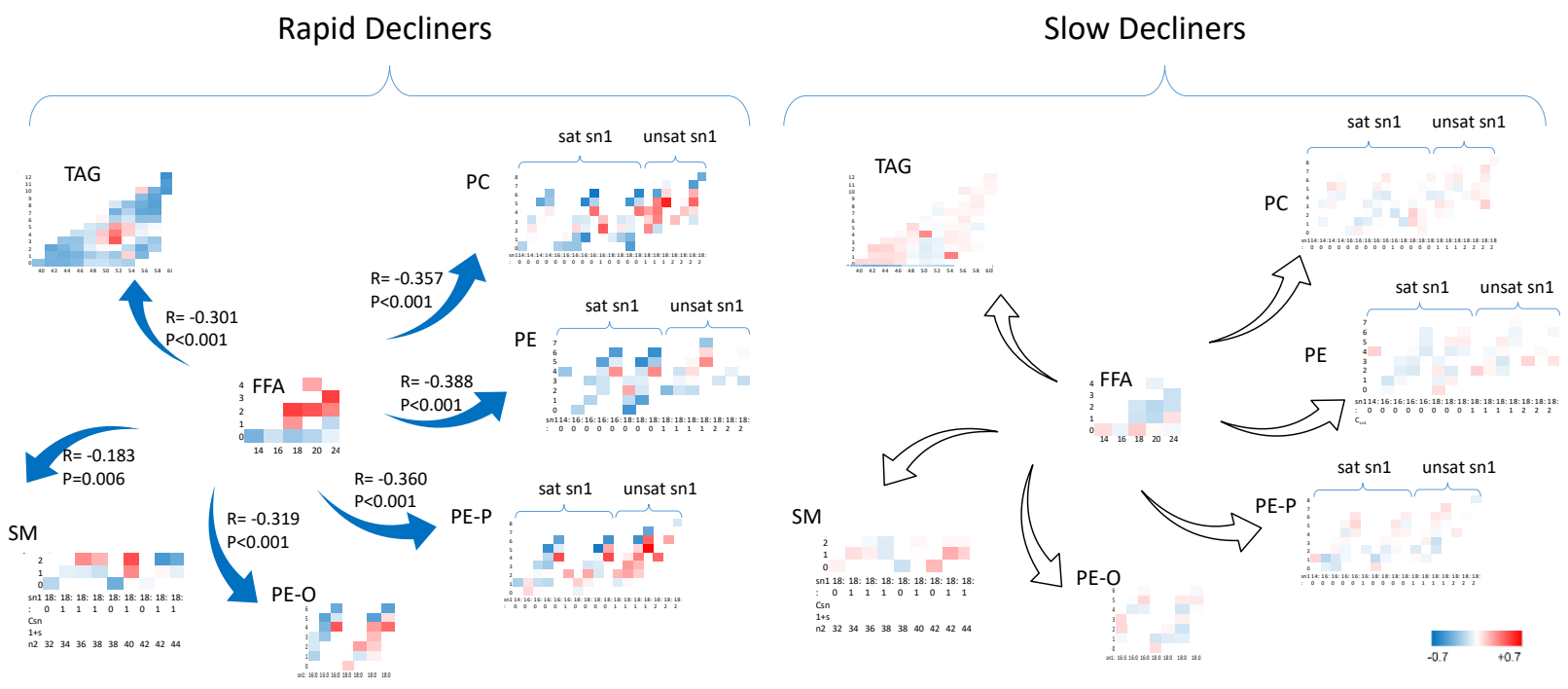

\section{B) eGFR $60-89 \mathrm{~mL} / \mathrm{min} / 1.73 \mathrm{~m} 2$}
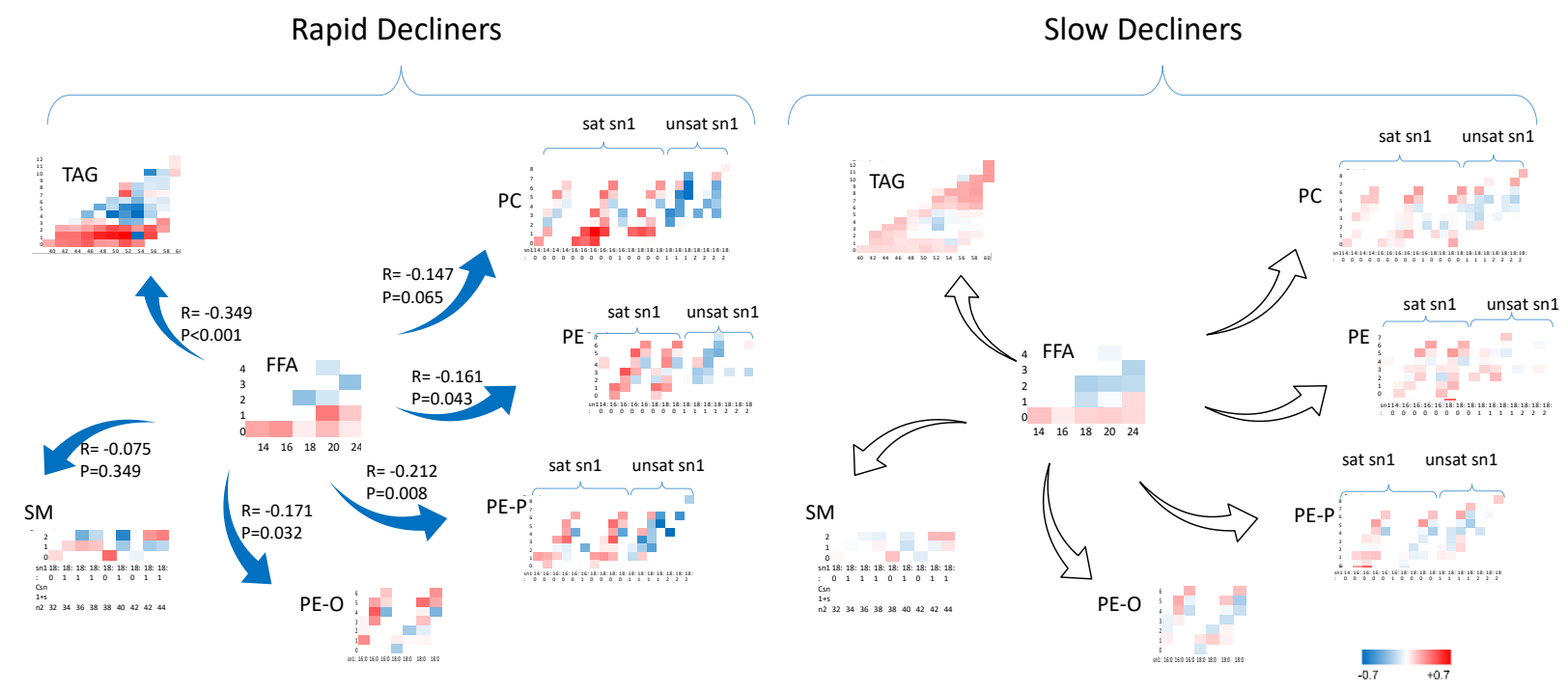

sFig 8. Differential abundance of various lipids during rapid versus slow eGFR decline by eGFR categories. A: In rapid decliners with baseline eGFR>90 mL/min/1.73m2 higher abundance of unsaturated FFAs, and unsaturated phospholipids at sn1 carbon, low abundance of saturated phospholipids at sn1 carbon and inverse correlations between unsaturated FFAs and saturated phospholipids at sn1 suggest shift of unsaturated FFAs in construct of unsaturated phospholipids. B: In rapid decliners with baseline eGFR of $60-89 \mathrm{~mL} / \mathrm{min} / 1.73 \mathrm{~m}^{2}$, relatively higher abundance of saturated FFAs, saturated phospholipids at sn1 carbon, lower unsaturated 
phospholipids at sn1 carbon, and inverse correlations between unsaturated FFAs and saturated PCs at sn1 suggest dominant shift of saturated FFAs in construct of saturated PCs at sn1 carbon. The $X$ axis of each plot is carbon number and the $Y$ axis is number of double bonds for the corresponding lipid class. Values are z-score mean standardized values for each lipid and $\mathrm{R}$ is the partial correlation between unsaturated FFA and unsaturated complex lipids adjusted for eGFR and albumin-creatinine ratio. AC, acylcarnitine; FFA, free fatty acid; PC, phosphatidylcholine; PE, phosphatidylethanolamine; SM, sphingomyelin; sat sn1, saturated acyl chain at sn1 carbon; TAG, triacylglycerol; unsat sn1, unsaturated acyl chain at sn1 carbon. 
sFig 9. System level lipids. A. Inter-class lipid factor correlations in the training and validation subsets reveal high direct correlation between saturated lipids and inverse correlation between saturated and unsaturated lipids. B. Classification power of lipid factors alone and when added to clinical variables (age, sex, diabetes duration, HbA1c, mean arterial pressure, eGFR, and $\log _{2}$ albumin-creatinine ratio) in the training set and replication in the validation set. $A C$, acylcarnitine; $\mathrm{Cl}$, confidence interval; uFFA, unsaturated free fatty acids; PC, phosphatidylcholine; PE, phosphatidylethanolamine; sPE-P, saturated PE with alkenyl ether substitute at sn-1 carbon; PE-O, PE with alkyl ether substitute at sn-1 carbon of the glycerol backbone; SM, sphingomyelin; TAG, triacylglycerol.

A)
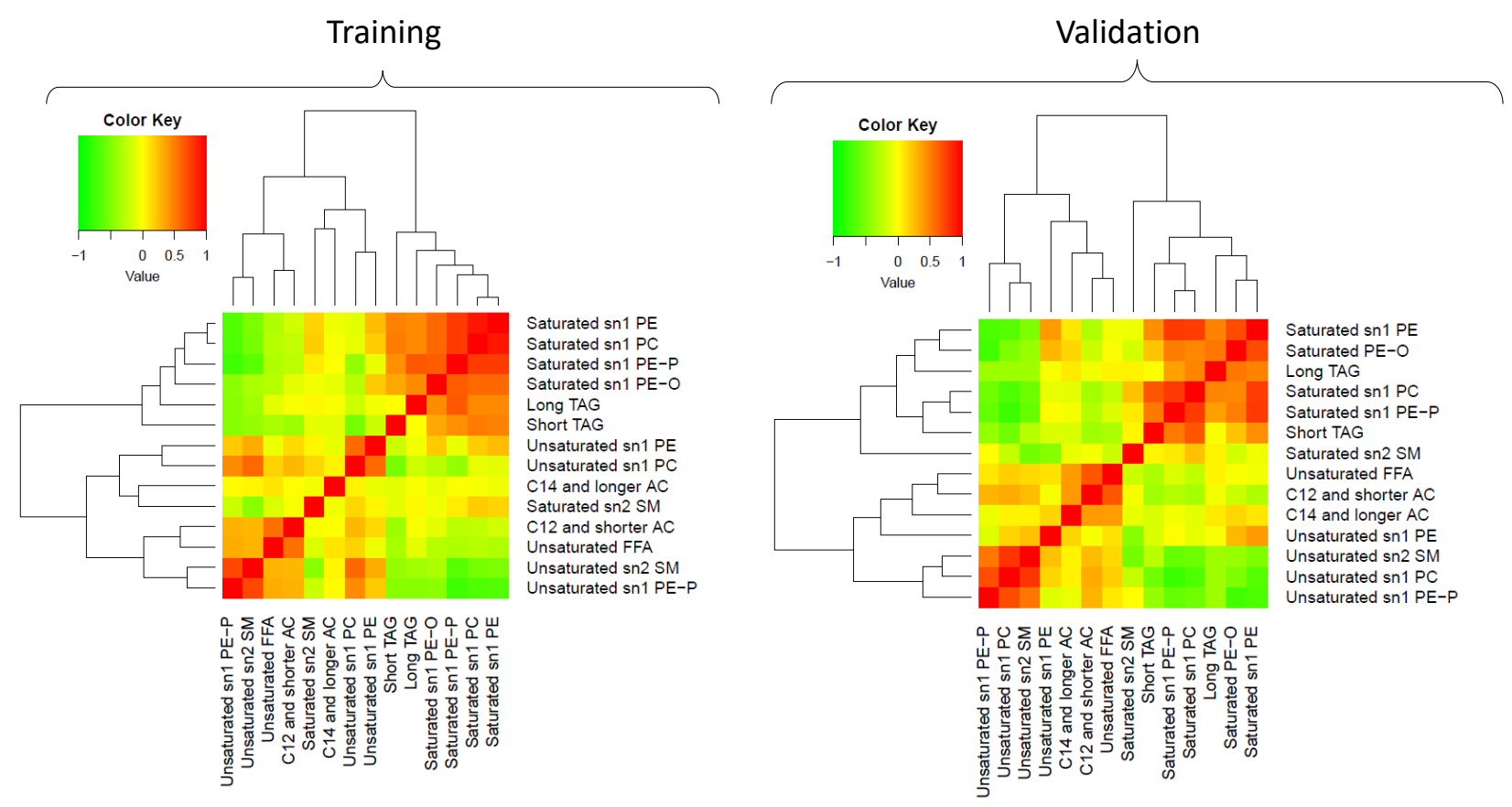

B)

\begin{tabular}{|l|c|c|}
\hline Panel & Training set & Validation set \\
\hline FFA(20:2) and PC(16:0/22:6) & & \\
\hline C-statistic (95\% Cl) & $0.610(0.555-0.664)$ & $0.679(0.624-0.735)$ \\
\hline uFFA, and sPE-P & & \\
\hline C-statistic (95\% Cl) & $0.707(0.652-0.763)$ & $0.697(0.642-0.752)$ \\
\hline Clinical & & \\
\hline C-statistic (95\% Cl) & $0.816(0.771-0.861)$ & $0.817(0.773-0.861)$ \\
\hline uFFA, and sPE-P + Clinical & & \\
\hline C-statistic (95\% Cl) & $0.841(0.799-0.883)$ & $0.836(0.793-0.879)$ \\
\hline
\end{tabular}


sFig 10: A) Alteration of fatty acids and complex lipids in rapid decliners of eGFR compared to slow decliners by eGFR categories. B) Comparing alterations of fatty acids and complex lipids in early stage (GFR $\geq 90 \mathrm{~mL} / \mathrm{min} / 1.73 \mathrm{~m}^{2}$ ) in rapid decliners in type 1 and type 2 diabetes. C) Proposed mechanisms of rapid eGFR decline involves diminished sensitivity of adipose tissue to insulin at early stage in insulin deficiency leading to diminished lipolysis and decreased circulating levels of TAGs, and relatively higher incorporation of unsaturated FFAs in construct of unsaturated phospholipids at the acyl chain in sn1 carbon of the glycerol backbone. With stage progression of CKD and CKD mediated higher insulin resistance, relative circulating levels of TAGs increase. In parallel, higher abundance of saturated FFAs due to insulin resistance and upregulation of de novo lipogenesis contributes to its higher incorporation in construct of saturated phospholipids at the acyl chain in sn1 carbon position of the glycerol backbone.

A) Comparing lipid alterations in rapid decliners by eGFR categories

\begin{tabular}{lccc}
\hline Lipids & $\begin{array}{c}\text { GFR } \mathbf{9 0} \\
\mathbf{m L} / \mathbf{m i n}\end{array}$ & $\begin{array}{c}\text { GFR 60-90 } \\
\text { mL/min }\end{array}$ & $\begin{array}{c}\text { GFR }<60 \\
\text { mL/min }\end{array}$ \\
\hline Saturated FFAs & $\downarrow$ & $\leftrightarrow$ or $\uparrow$ & $\leftrightarrow$ or $\uparrow$ \\
\hline Unsaturated FFAs & $\uparrow$ & $\leftrightarrow$ or $\downarrow$ & $\leftrightarrow$ or $\downarrow$ \\
\hline Long Chain AC & $\leftrightarrow$ or $\uparrow$ & $\leftrightarrow$ or $\uparrow$ & $\leftrightarrow$ or $\uparrow$ \\
\hline Shorter TAGs & $\downarrow$ & $\uparrow$ & $\uparrow$ \\
\hline Longer TAGs & $\downarrow$ & $\uparrow$ & $\uparrow$ \\
\hline Saturated PCs & $\downarrow$ & $\uparrow$ & $\uparrow$ \\
\hline Unsaturated PCs & $\uparrow$ & $\downarrow$ & $\downarrow$ \\
\hline Saturated PEs & $\downarrow$ & $\uparrow$ & $\uparrow$ \\
\hline Saturated PE-O & $\downarrow$ & $\uparrow$ & $\uparrow$ \\
\hline Saturated PE-P & $\downarrow$ & $\uparrow$ & $\uparrow$ \\
\hline Unsaturated PE-P & $\uparrow$ & $\downarrow$ & $\downarrow$ \\
\hline
\end{tabular}

B) Comparing lipid alterations in fast progressors with baseline GFR $\geq 90$ $\mathrm{mL} / \mathrm{min}$ by type of diabetes

\begin{tabular}{lll}
\hline Lipids & Type & Type \\
& 1 DM & 2 DM
\end{tabular}

\begin{tabular}{lcc}
\hline Saturated FFA & $\downarrow$ & $\uparrow$ \\
\hline Unsaturated FFA & $\uparrow$ & $\downarrow$ \\
\hline Long Chain AC & $\leftrightarrow$ or $\uparrow$ & $\downarrow$ \\
\hline Shorter Chain TAGs & $\leftrightarrow$ or $\downarrow$ & $\downarrow$ \\
\hline Longer Chain TAGs & $\downarrow$ & $\uparrow$ \\
\hline Unsaturated PEs & $\uparrow$ & $\uparrow$ \\
\hline
\end{tabular}

C) Proposed lipidomic mechanisms of DKD progression in type 1 diabetes eGFR $\geq 90 \mathrm{~mL} / \mathrm{min}:$
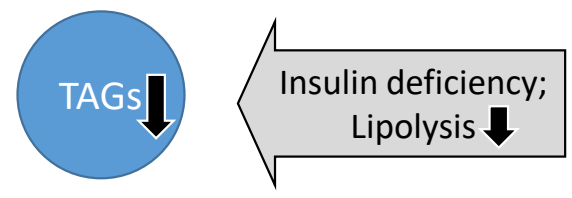

eGFR $60-90 \mathrm{~mL} / \mathrm{min}$ :
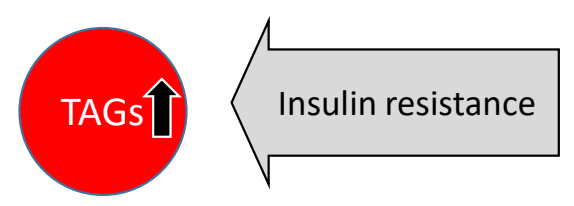
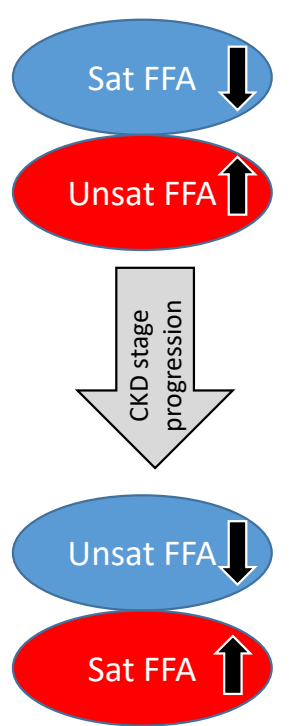

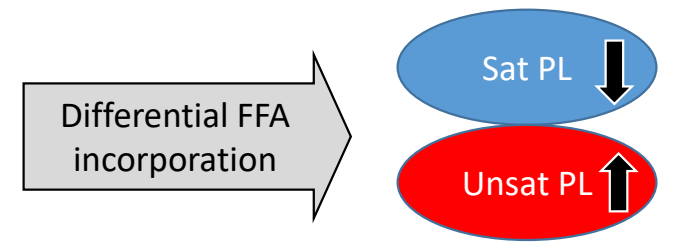

